\title{
CORONAVÍRUS: EXPLORANDO O TEMA ATRAVÉS DA BIBLIOMETRIA
}

\author{
Eduardo Amadeu Dutra Moresi ${ }^{1}$ e Isabel Pinho ${ }^{2}$ \\ 1Universidade Católica de Brasília, Brasil. moresi@ucb.br; ²Universidade de Aveiro, Portugal. isabelpinho@ua.pt
}

\begin{abstract}
Resumo. O coronavírus se tornou um tema transversal para várias comunidades: científicas, políticas, sociais e econômicas. Em particular, tem sido divulgado que o COVID-19 surgiu no final de 2019 e representa uma séria ameaça global, com impacto na saúde pública, na busca de vacina e tratamento, em estratégias para impedir a propagação de infecções, em viagens, em negócios, no trabalho e comportamentos sociais. Para coletar informações científicas relevantes sobre o que é coronavírus, este artigo apresenta uma análise bibliométrica dos dados coletados na base Scopus. Foi realizada uma análise exploratória, com base nos metadados de 20.438 documentos, de 1951 a março/2020. Os dados foram tratados com o apoio do CiteSpace que nos permitiu obter uma visão global da evolução das publicações, a rede de cocitação, as comunidades temáticas e os documentos mais citados. Os resultados obtidos podem ser um sólido ponto de partida para futuras explorações. Assim, a bibliometria pode contribuir para garantir a qualidade da informação, condição indispensável para o desenvolvimento científico de um determinado tema. Além dessas informações, jornalistas e formuladores de políticas e gerentes educacionais e organizacionais também devem considerar a bibliometria como uma ferramenta de tomada de decisão baseada em evidências.
\end{abstract}

Palavras-chave: Coronavírus; Processos de Conhecimento; Bibliometria; Metadados; Análise de Cocitação.

\section{CORONAVIRUS: EXPLORING THE SUBJECT THROUGH BIBLIOMETRICS}

Abstract. Coronavirus has become a crosscutting theme for several communities: scientific, political, social, and economic. In particular, COVID-19 emerged at the end of 2019 and represents a serious global threat, with an impact on public health, in the search for vaccine and treatment, in strategies to prevent the spread of infection, in travel, in business, at work and social behaviors. To collect relevant scientific information about what is a coronavirus, this article presents a bibliometric analysis of data collected from Scopus. An exploratory analysis was carried out, based on the metadata of 20,438 documents, from 1951 to March 2020. The data were treated with the support of CiteSpace that allowed us to obtain a global view of the evolution of publications, the co-citation network, cluster subjects, and the most cited documents. The results obtained can be a solid starting point for further exploration. Thus, bibliometrics can contribute to the guarantee of information quality, an indispensable condition for the scientific development of a given theme. In addition to these information users, journalists and policymakers and educational and organizational managers should also consider bibliometrics as an evidence-based decision-making tool.

Keywords: Coronavirus; Knowledge Processes; Bibliometric; Metadata; Co-Citation Analysis.

\section{INTRODUÇÃO}

O surto de doença por coronavírus em dezembro de 2019, colocou o tema sob o foco da necessidade de informação de qualidade para um diverso leque de utilizadores e produtores de informação. A necessidade de basear a tomada de decisão em evidência é prioritária para que o impacto dessa doença seja minimizado. 
A síndrome respiratória aguda grave, coronavírus 2 (SARS-CoV-2) e a nova doença de coronavírus (COVID-19), têm sido motivo de grande preocupação para a comunidade de saúde em geral, mas principalmente para médicos especializados em doenças infeciosas e medicina pulmonar e de cuidados intensivos (Yan et al, 2020).

A comunidade científica está mobilizada para uma rápida e eficaz apresentação de soluções. Não só em nível científico, mas também em nível da cidadania responsável, onde a informação se torna um recurso relevante para diversos interessados. Políticos e gestores de organizações são chamados a tomar decisões que afetam o quotidiano, a economia e principalmente a saúde de populações em todos os níveis. Em nível prático, as decisões sobre como, por exemplo, aplicar a quarentena requerem uma base sólida baseada nas melhores evidências disponíveis. Uma pergunta é transversal a todos estes interessados em recolher e usar a informação: quais as frentes de pesquisa sobre o coronavírus?

Este tipo de questão apela à mobilização de grande quantidade de informação que requer competência para a sua seleção, análise e posterior disseminação pelos diversos segmentos de utilizadores. Este é o campo da gestão de conhecimento, que pode ajudar a encontrar, a usar informação disponível e a mobilizar o conhecimento existente de modo eficaz (Rego et al., 2009). Com vista a simplificar este complexo fenômeno é possível considerar uma cadeia de quatro processos de conhecimento: a) aquisição de conhecimento; b) criação de conhecimento; c) transferência de conhecimento; e d) partilha de conhecimento (Pinho, 2012; Pinho e Diogo, 2018). Considerando o conhecimento como um recurso intangível, interessa compreender como os fluxos de conhecimento perpassam os diversos processos de conhecimento (ver Figura 1).

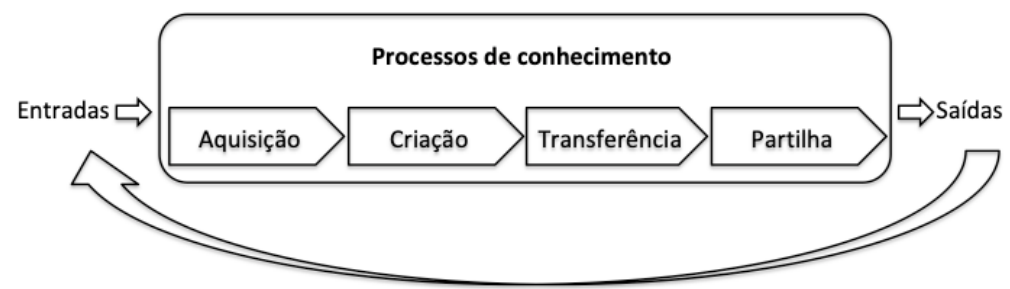

Figura 1. Estrutura da cadeia de processos de conhecimento (Pinho, 2012; Pinho e Diogo, 2018)

No caso deste artigo, a tarefa situa-se no processo de aquisição de conhecimento, que se refere à busca, identificação, seleção, recolha, organização e mapeamento de informação. Como estratégia de investigação decidimos usar a bibliometria como uma ferramenta de exploração do tema, com vista a responder à questão inicial. A análise bibliométrica refere- 
se à combinação de diferentes estruturas, ferramentas e métodos estudar e analisar citações das publicações, permitindo obter a estrutura intelectual de uma disciplina ou de um tema (Hood e Wilson, 2001; Leydesdorff e Bornmann, 2016).

Após selecionarmos os artigos relevantes (no máximo de 100 artigos), estes serão objeto de uma análise de conteúdo (Bardin, 2004) para responder a algumas questões que irão emergir.

Este artigo exploratório será ponto de partida para outros artigos que irão resultar de futuras etapas de aprofundamento do tema poderão explorar potencialidades da bibliometria para responder a questões práticas, como por exemplo: Quais as equipas de investigação que poderão trabalhar de forma colaborativa para acelerar o processo de criação de conhecimento e transferência de conhecimento com impacto no combate ao Coronavírus?

Deste modo, iremos aproveitar as potencialidades da investigação mista, considerando as abordagens quantitativas e qualitativas como complementares, para a integração do conhecimento.

\section{METODOLOGIA}

O mapeamento científico utiliza métodos bibliométricos para examinar como as disciplinas, campos, especialidades e documentos individuais estão relacionados entre si. Produz uma representação espacial dos achados análogos aos mapas geográficos (Calero-Medina \& van Leeuwen, 2012; Small, 1999). O mapeamento científico é uma combinação de classificação e visualização (Boyack \& Klavans, 2014). O objetivo é criar uma representação da estrutura da área de pesquisa, particionando elementos (documentos, autores, periódicos, palavras) em diferentes grupos. A visualização é então usada para criar uma representação visual da classificação que emerge.

Este estudo adotou o método de revisão bibliométrica, porque este tipo de análise fornece uma ferramenta útil para inferir conhecimentos de um corpo de literatura e interpretar a evolução, distribuição e desenvolvimento de pesquisas em um determinado campo (Zupic \& Cater, 2014; Soriano, Álvarez \& Valdés, 2018). Com base na análise da cocitação, a cientometria pode ajudar os pesquisadores a entender o mapeamento do conhecimento dos 
campos científicos e rastrear fronteiras de desenvolvimento (Soriano, Álvarez \& Valdés, 2018). O diagrama de fluxo da seleção e análise de dados é ilustrado na Figura 2.

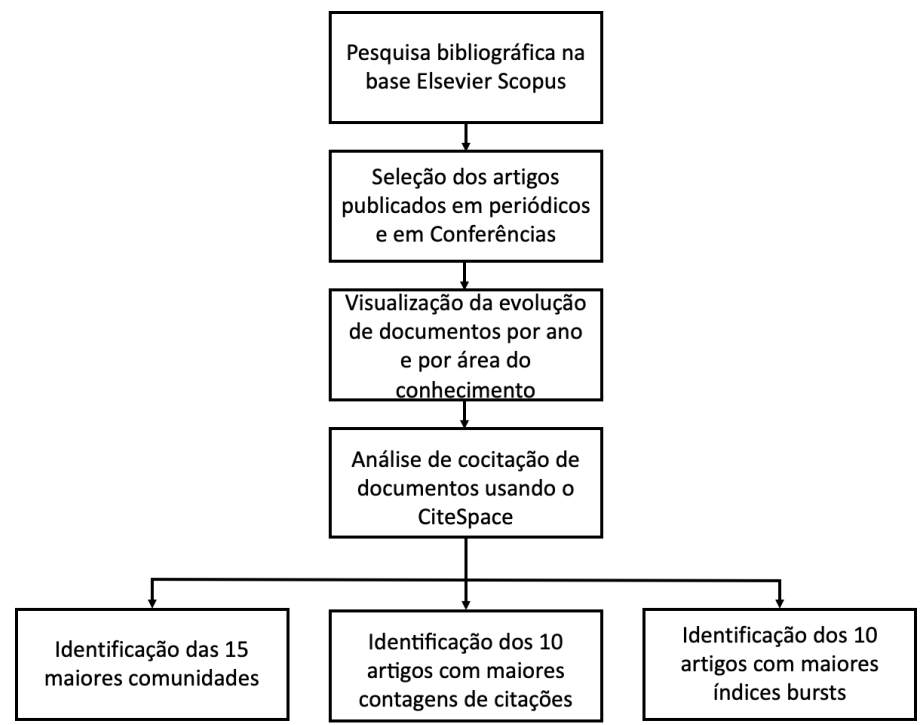

Figura 2. Fluxo da análise bibliométrica da pesquisa bibliográfica sobre Coronavírus.

Consequentemente, muitas técnicas de mapeamento científico foram geradas para analisar redes de referências citadas com base em registros bibliográficos. O CiteSpace representa um software gratuito e popular, projetado especificamente para explorar as tendências emergentes e mudanças importantes em várias áreas de pesquisa (Hou et al, 2019; Cui, Mou \& Liu, 2018; Liu, Liu, \& Zhang, 2014). Ele utiliza um conjunto de registros bibliográficos como entrada e delineia a estrutura intelectual do domínio do conhecimento por meio de redes de cocitação de documentos e algoritmos de localização (Chen, 2019). Portanto, no presente estudo, o CiteSpace foi escolhido como a ferramenta analítica visual para analisar a literatura existente sobre tema da Coronavirus.

A partir de um levantamento preliminar dos termos associado ao coronavírus, foi realizada uma pesquisa na base Scopus utilizando a expressão de busca: coronavirus OR "Corona virus" OR "2019-nCoV" OR "SADS-CoV" OR "SARS-CoV" OR "MERS-CoV" OR "Severe Acute Respiratory Syndrome" OR "Middle East Respiratory Syndrome" OR "covid-19". Foram recuperados 20.438 documentos, sendo 19.482 artigos publicados em periódicos e 956 em eventos científicos, cobrindo o período de 1951 a 2020. A Figura 3 apresenta a evolução das publicações por ano. Observa-se que o pico de publicações ocorreu em 2004, com 1.533 documentos. No período de 2006 a 2011 houve um decréscimo significativo, voltando a crescer até alcançar 934 documentos em 2015. Em 2019 foram, até o momento, 
publicados 855 documentos. Em 2020, devido ao crescimento de pessoas infectadas, inicialmente na China, e, posteriormente, em diversos países, já foram publicados 276 documentos apenas em dois meses. Isso indica, que 2020 há a expetativa de um crescimento significativo no número de publicações.

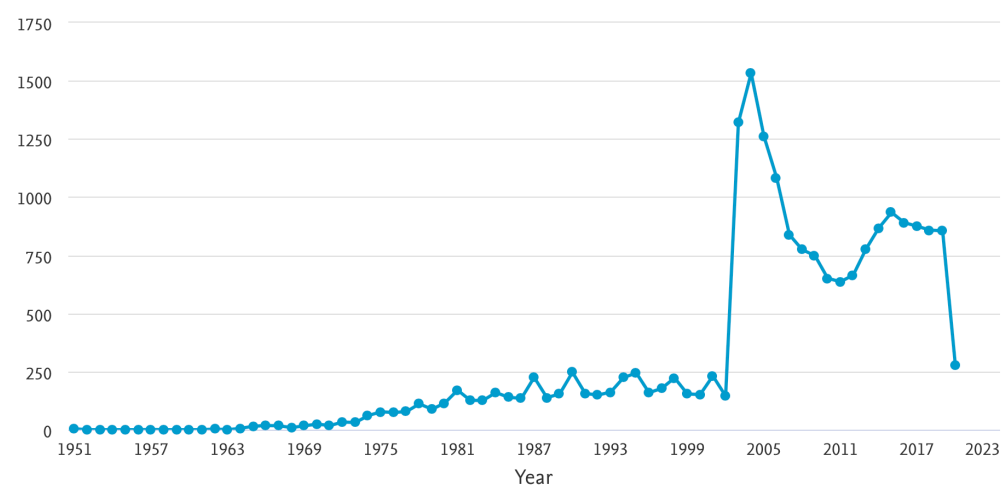

Figura 3. Evolução das publicações por ano.

Outro ponto interessante a destacar é o percentual de documentos por área do conhecimento, que é mostrado na Figura 4. Observa-se que os maiores percentuais são de Medicina (30,7\%), Imunologia e Microbiologia (22,0\%), Bioquímica, Genética e Biologia Molecular $(14,0 \%)$, Veterinária $(8,5 \%)$ e Agricultura e Ciências Biológicas (8,3\%). Esse resultado mostra que as publicações são da área de saúde e afins.

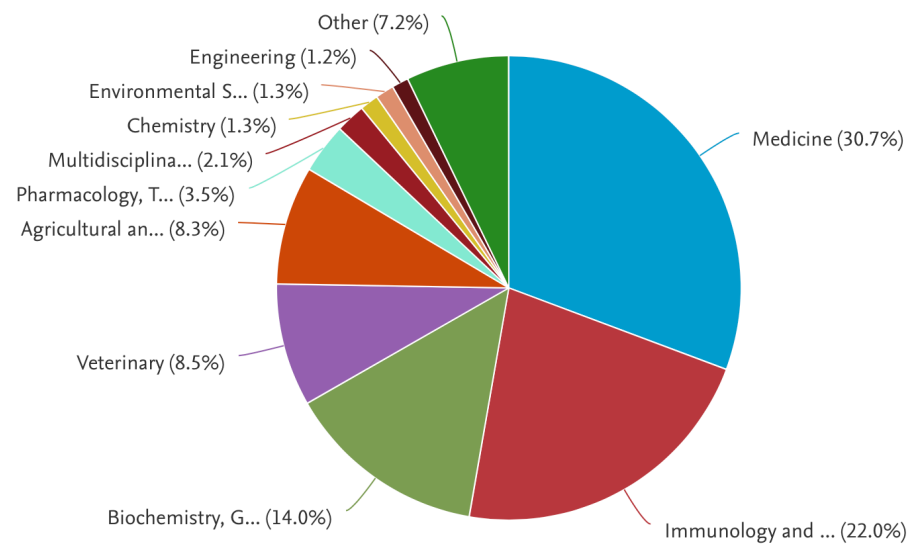

Figura 4. Distribuição de documentos por área do conhecimento.

A análise bibliométrica seguiu os procedimentos apresentados no manual do CiteSpace (Chen, 2014). O CiteSpace suporta análise de cocitação de autor, análise de cocitação de documento e análise de coocorrência de palavras-chave, modelando e visualizando redes e mapas. Nos mapas de conhecimento de visualização, existem nós e ligações que representam elementos ou unidades de análise (ou seja, autores, documentos, instituições e 
países) e relações ou tipos de análise (ou seja, coocorrência de palavras-chave, cocitação de documentos, coautoria, etc). Para revelar os padrões e tendências de pesquisa sobre Coronavírus, foram realizadas análises de cocitação de referências bibliográficas, com os nós sendo as referências e as arestas as ligações de cocitação.

Alguns parâmetros no CiteSpace devem ser adequadamente definidos de acordo com os objetivos da pesquisa:

- o valor da escala de tempo foi definido como 1, o que significa que todo o intervalo de tempo foi dividido em várias fatias de 1 ano para processamento de dados;

- para formar as redes finais, foram seguidos os critérios de seleção do nó, "Top 50 por slice" e "Interpolação do limite". "Top 50 por slice" significa que os 50 itens mais citados ou ocorridos de cada ano serão selecionados para construir uma rede. Quando o número de itens não for grande o suficiente para apresentar uma rede, a Interpolação do limite é usada.

Além disso, as métricas utilizadas neste estudo foram a cocitação por explosão (Co-citation Bursts), que fornece evidências de que uma publicação específica está associada a um aumento nas citações (Chen, 2019). O CiteSpace rotula cada cluster com base em três algoritmos especializados - frequência inversa de documentos (TF * IDF), testes de probabilidade de log (LLR) e testes de informação mútua (MI). O LLR geralmente fornece o melhor resultado em termos de exclusividade e cobertura (Chen, Ibekwe-SanJuan, \& Hou, 2010). Assim, neste estudo, a LLR foi empregada.

Para garantir a qualidade da análise de cluster, dois indicadores importantes devem ser verificados para medir as propriedades estruturais da rede.

A modularidade $Q$ representa a extensão em que uma rede pode ser separada em múltiplos componentes. Uma alta modularidade pode indicar uma rede bem estruturada, mas redes com pontuações de modularidade de 1 ou muito próximas de 1 podem refletir o comportamento ou preferência de citação de um único artigo, portanto, é menos representativo (Chen, 2019). O valor da silhueta representa o nível de incerteza ao interpretar a natureza do cluster, isto é, a homogeneidade de um cluster. Seu valor varia entre - 1 e 1. Quanto mais próximo o valor de 1, mais consistentes são os membros do cluster. Neste estudo, espera-se que a rotulagem do cluster seja mais direta, com um valor de silhueta superior a 0,5. 


\section{RESULTADOS DA PESQUISA}

O coronavírus pertence a uma grande família de vírus que geralmente causam doenças leves a moderadas do trato respiratório superior, como o resfriado comum. No século XXI, os surtos de coronavírus surgiram pela transmissão por animais, causando doenças graves e problemas em escala global (Gorbalenya et al., 2020).

Existem centenas de coronavírus, a maioria dos quais circula entre animais, incluindo porcos, camelos, morcegos e gatos.

Às vezes, esses vírus são transmitidos para os humanos - chamados de evento de transbordamento - e podem causar doenças (National Institute of Allergy and Infectious Diseases, 2020). Três dos coronavírus podem ter resultados mais sérios nas pessoas, e essas doenças são: a SARS (síndrome respiratória aguda grave) que surgiu no final de 2002 e desapareceu em 2004; a MERS (síndrome respiratória do Oriente Médio), que surgiu em 2012 e permanece em circulação nos camelos; COVID-19, que foi divulgado como tendo surgido em dezembro de 2019 da China e um esforço global está em andamento para conter seu spread. O COVID-19 é causado pelo coronavírus conhecido como SARS-CoV-2 (Liu et al., 2020; Malta et al., 2020).

A análise dos dados recolhidos (20.438 registros) baseou-se na rede de cocitação, que permitiu obter uma estrutura conceptual de pesquisa (Chen et al., 2010). A rede gerada, que é apresentada na Figura 5, possui 3.225 nós e 7.164 arestas.

Em seguida, foi executada a obtenção das comunidades (clusters) utilizando o algoritmo Log-likelihood Ratio (Chen, Ibekwe-SanJuan \& Hou, 2010) para alocar cada citação em uma comunidade (Chen, 2019, p. 52), obtendo a Modularidade $Q$ de 0,8756 e 395 comunidades. A barra superior da Figura 5 possui uma variação de cores, da esquerda para a direita, de magenta até amarelo. Essas cores identificam os anos das publicações: de 1971 (magenta) a 2020 (amarelo). 


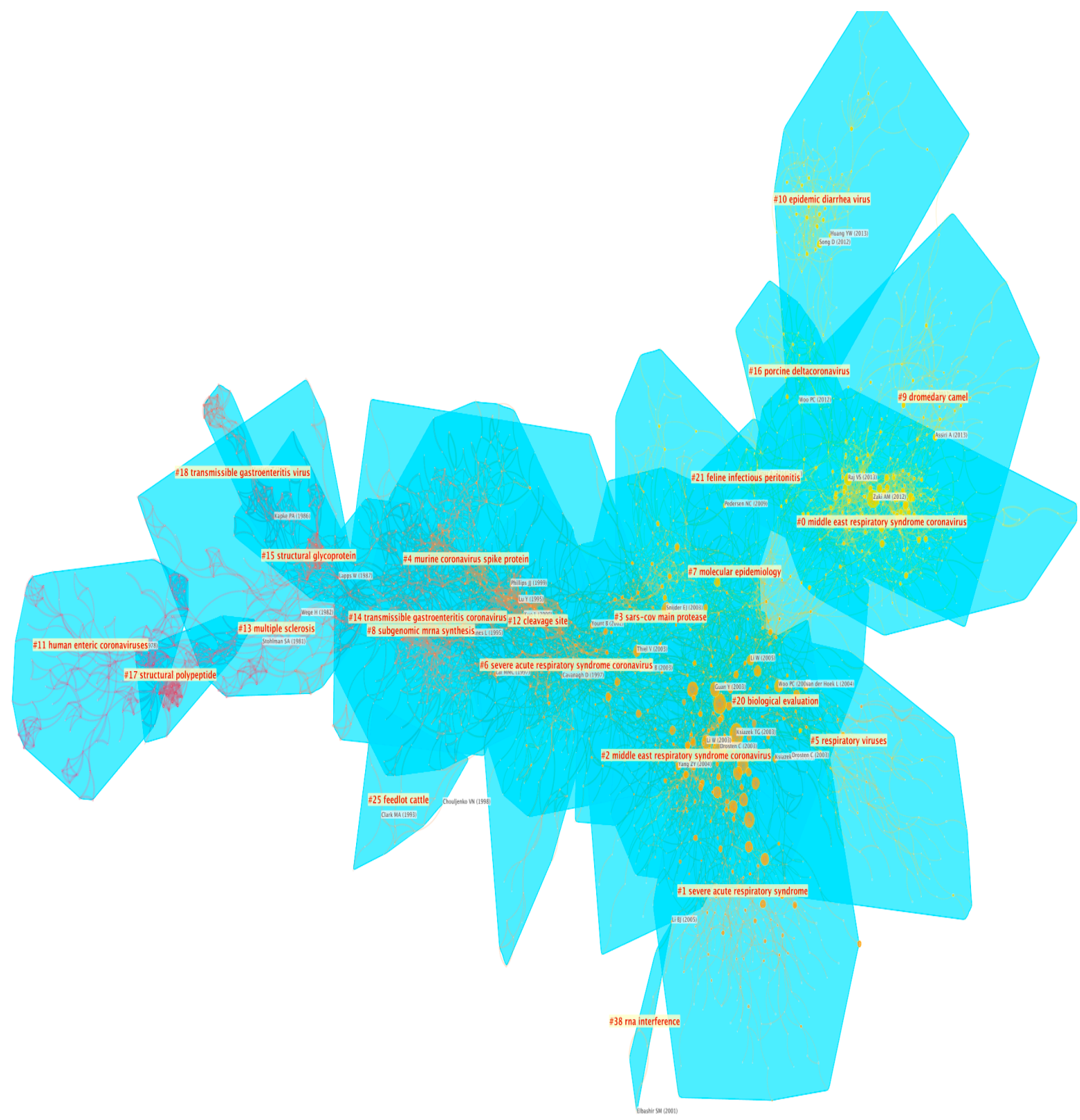

Figura 5. Rede de cocitação de documentos gerada pelo CiteSpace a partir da pesquisa bibliográfica realizada na base Scopus.

A Tabela 1 apresenta uma síntese das 15 maiores comunidades, incluindo o rótulo da comunidade, o número de documentos, a silhueta e o ano médio. $O$ valor da silhueta de uma comunidade mede a qualidade de sua configuração. Seu valor varia entre -1 e 1.0 valor mais alto representa uma solução perfeita (Chen et al., 2010). Observa-se que todas as comunidades apresentam valores de silhueta superiores a 0,8 . 
Tabela 1. Síntese das 15 maiores comunidades utilizando o CiteSpace.

\begin{tabular}{lccc}
\hline Comunidade & Nr documentos & Silhueta & Ano médio \\
\hline $\begin{array}{l}\text { 0. middle east respiratory syndrome } \\
\text { coronavirus }\end{array}$ & 276 & 0,884 & 2014 \\
1. severe acute respiratory syndrome & 228 & 0.838 & 2004 \\
coronavirus & 210 & 0,804 & 1998 \\
2. murine coronavirus spike protein & 173 & 0,788 & 2006 \\
3. sars-cov main protease & 156 & 0,841 & 2006 \\
4. human coronaviruses & 134 & 0,943 & 2003 \\
5. severe acute respiratory syndrome & 133 & 0,912 & 1992 \\
6. new transcriptional unit & 122 & 0,963 & 2014 \\
7. epidemic diarrhea virus & 106 & 0,948 & 1981 \\
8. human enteric coronaviruses & 103 & 0,964 & 2013 \\
9. dromedary camel & 89 & 0,983 & 1975 \\
10. other species & 75 & 0,945 & 1996 \\
11. cleavage site & 71 & 0,969 & 1994 \\
12. neurotropic coronavirus & 64 & 0,948 & 2007 \\
13. respiratory viral panel & 56 & 0,984 & 1985 \\
14. transmissible gastroenteritis virus & & & \\
\hline
\end{tabular}

Fonte: CiteSpace - 2020 .

A Tabela 2 apresenta os 14 artigos com as maiores contagens de citações. Observa-se que 14 artigos são do ano de 2003. Esse fato está relacionado ao coronavírus (SARS-CoV) que foi descoberto em associação com casos de síndrome respiratória aguda grave (SARS), em março de 2003 (Rota et al., 2003). Nove estudos trataram da relação entre o coronavírus e a SARS (Drosten et al., 2003; Guan et al., 2003; Ksiazek et al., 2003; Lee et al., 2003; Li et al., 2003; Marra et al., 2003; Peiris et al., 2003; Poutanen et al., 2003; Rota et al., 2003). Um estudo focou no coronavírus HCov-EMC (Zaki et al., 2012) e outro abordou sobre a progressão temporal das alterações clínicas, radiológicas e virológicas em um surto comunitário de SARS (Peiris et al, 2003b).

Tabela 2. Os 10 documentos com as maiores contagens de citações.

\begin{tabular}{lcc}
\hline Referência citada & Contagem de Citações & Comunidade \\
\hline Drosten et al (2003) & 997 & 2. murine coronavirus spike protein \\
Ksiazek et al (2003) & 966 & 2. murine coronavirus spike protein \\
Rota et al (2003) & 811 & 2. murine coronavirus spike protein \\
Zaki et al (2012) & 720 & 4. human coronaviruses \\
Lee et al (2003) & 636 & 1. severe acute respiratory syndrome coronavirus \\
Peiris et al (2003a) & 573 & 2. murine coronavirus spike protein \\
Marra et al (2003) & 522 & 2. murine coronavirus spike protein \\
Guan et al (2003) & 435 & 3. sars-cov main protease \\
Li et al (2003) & 432 & 3. sars-cov main protease \\
Poutanen et al & 411 & 1. severe acute respiratory syndrome coronavirus \\
(2003) & 380 & \#1 severe acute respiratory syndrome coronavirus \\
Tsang et al (2003) & 373 & \#1 severe acute respiratory syndrome coronavirus \\
Peiris et al (2003b) & 337 & \#3 sars-cov main protease \\
Snijder et al (2003) & 322 & \#1 severe acute respiratory syndrome coronavirus \\
Booth et al (2003) & 316 & \#1 severe acute respiratory syndrome coronavirus \\
Kuiken et al (2003) &
\end{tabular}

Fonte: CiteSpace - 2020 . 
A Tabela 3 apresenta as áreas onde houveram aumento na incidência de publicações, utilizando a funcionalidade Bursts do CiteSpace (Chen, 2019). Observa-se que 14 estudos trataram da relação entre o coronavírus e a SARS (Drosten et al., 2003; Ksiazek et al., 2003; Lee et al., 2003; Marra et al., 2003; Poutanen et al., 2003; Raj et al., 2013; Rota et al., 2003; Tsang et al., 2003; Zaki et al., 2012), entre o coronavírus e a MERS (Assiri et al., 2013b).

Tabela 3. Os 10 documentos com os maiores índices Bursts.

\begin{tabular}{|c|c|c|}
\hline Referência citada & $\begin{array}{l}\text { Índice } \\
\text { Bursts }\end{array}$ & Comunidade \\
\hline Zaki et al (2012) & 272,31 & 4. human coronaviruses \\
\hline Drosten et al (2003) & 163,43 & 2. murine coronavirus spike protein \\
\hline Ksiazek et al (2003) & 161,96 & 2. murine coronavirus spike protein \\
\hline Lee et al (2003) & 158,93 & 1. severe acute respiratory syndrome coronavirus \\
\hline Rota et al (2003) & 146,90 & 2. murine coronavirus spike protein \\
\hline Poutanen et al (2003) & 107,73 & 1. severe acute respiratory syndrome coronavirus \\
\hline Tsang et al (2003) & 102,76 & 1. severe acute respiratory syndrome coronavirus \\
\hline Raj et al (2013) & 99,9 & 3. sars-cov main protease \\
\hline Marra et al (2003) & 98,3 & 2. murine coronavirus spike protein \\
\hline Assiri et al (2013a) & 96,74 & 3. sars-cov main protease \\
\hline Assiri et al (2013b) & 89,52 & \#0 middle east respiratory syndrome coronavirus \\
\hline Li et al (2005) & 85,14 & \#4 human coronaviruses \\
\hline Peiris et al (2003a) & 80,16 & \#1 severe acute respiratory syndrome coronavirus \\
\hline Li et al (2003) & 78,38 & \#1 severe acute respiratory syndrome coronavirus \\
\hline Lau et al (2005) & 77,83 & \#4 human coronaviruses \\
\hline
\end{tabular}

Fonte: CiteSpace - 2020.

Observa-se que dez referências estão entre as mais cocitadas e com os maiores índices de Bursts, havendo a maior concentração em publicações do ano de 2003. Este resultado é coerente, devido ao surto de SARS que ocorreu no final do ano de 2002. A frente de pesquisa mais recente se refere ao coronavírus humano, que possui o médio de publicações em 2006. Contudo, o artigo mais relevante é de 2012.

\section{CONCLUSÕES}

O mapeamento do tema através das variadas vertentes de visualização dos dados obtidos é uma fase importante para que se possa decidir sobre o foco a ser dado em uma futura revisão integrativa sobre a Coronavírus.A metodologia é consistente, pois possibilita uma análise abrangente dos documentos publicados em um período de tempo. Contudo, a definição da expressão de busca é um ponto crítico, pois implicará diretamente nos documentos que serão recuperados em uma pesquisa bibliográfica. Contudo, a evolução dos quantitativos de documentos por ano permite analisar a evolução do tema ao longo do período em análise. 
Outro ponto a destacar, é que este Trata-se de um tema que interessa a diversas áreas do conhecimento, mas que se vai construindo como área de investigação/pesquisa com teoria e prática estruturada. Autores e publicações relevantes são cocitados criando redes de conhecimento que consolidam este tema.

estudo bibliométrico revelou que os anos de 2003 e de 2012 marcaram dois pontos de crescimento nas publicações sobre o coronavírus. Em ambos os casos, o interesse pelo tema teve origem na ocorrência de casos detectados e reportados pela Organização Mundial da Saúde. Considerando o momento atual, devido a um novo surto do coronavírus com propagação em nível mundial, há a expectativa de um novo crescimento nas publicações sobre o tema devido ao comprometimento dos cientistas em apresentar soluções para a pandemia.

Como prosseguimento da investigação, sugerimos que um novo recorte seja realizado com foco nas publicações dos últimos 3 anos, de 2017 a 2020, para que sejam identificadas as frentes de pesquisa mais aderentes ao momento atual.

\section{REFERÊNCIAS}

Bardin, L. (2004). Análise de Conteúdo (R. Luís \& A. Pinheiro, Trans.). Lisboa: Edições 70.

Chen, C. (2019). How to use CiteSpace. Victoria, British Columbia, CA: Leanpub.

Chen, C., Ibekwe-SanJuan, F. e Hou, J. (2010). The structure and dynamics of cocitation clusters: A multipleperspective cocitation analysis. Journal of the American Society for Information Science and Technology, 61(7), 1386-1409.

Drosten, C., Günther, S., Preiser, W., van der Werf, S., Brodt, H.-R., Becker, S., Rabenau, H., Panning, M., Kolesnikova, L., Fouchier, R. A. M., Berger, A., Burguière, A.-M., Cinatl, J., Eickmann, M., Escriou, N., Grywna, K., Kramme, S., Manuguerra, J.-C., Müller, S., Rickerts, V., Stürmer, M., Vieth, S., Klenk, H.-D., Osterhaus, A. D. M. E., Schmitz, H. e Doerr, H. W. (2003). Identification of a Novel Coronavirus in Patients with Severe Acute Respiratory Syndrome. New England Journal of Medicine, 348(20), 1967-1976.

Gorbalenya, A. E., Baker, S. C., Baric, R. S., de Groot, R. J., Drosten, C., Gulyaeva, A. A., Haagmans, B. L., Lauber, C., Leontovich, A. M., Neuman, B. W., Penzar, D., Perlman, S., Poon, L. L. M., Samborskiy, D. V., Sidorov, I. A., Sola, I., Ziebuhr, J. e Coronaviridae Study Group of the International Committee on Taxonomy of, V. (2020). The species Severe acute respiratory syndrome-related coronavirus: classifying 2019-nCoV and naming it SARS-CoV-2. Nature Microbiology, 5(4), 536-544.

Guan, Y., Zheng, B. J., He, Y. Q., Liu, X. L., Zhuang, Z. X., Cheung, C. L., Luo, S. W., Li, P. H., Zhang, L. J., Guan, Y. J., Butt, K. M., Wong, K. L., Chan, K. W., Lim, W., Shortridge, K. F., Yuen, K. Y., Peiris, J. S. M. e Poon, L. L. M. (2003). Isolation and Characterization of Viruses Related to the SARS Coronavirus from Animals in Southern China. Science, 302(5643), 276-278.

Hood, W. W. e Wilson, C. S. (2001). The literature of bibliometrics, scientometrics, and informetrics. Scientometrics, 52(2), 291-314. 
Ksiazek, T. G., Erdman, D., Goldsmith, C. S., Zaki, S. R., Peret, T., Emery, S., Tong, S., Urbani, C., Comer, J. A., Lim, W., Rollin, P. E., Dowell, S. F., Ling, A.-E., Humphrey, C. D., Shieh, W.-J., Guarner, J., Paddock, C. D., Rota, P., Fields, B., DeRisi, J., Yang, J.-Y., Cox, N., Hughes, J. M., LeDuc, J. W., Bellini, W. J. e Anderson, L. J. (2003). A Novel Coronavirus Associated with Severe Acute Respiratory Syndrome. New England Journal of Medicine, 348(20), 1953-1966.

Lee, N., Hui, D., Wu, A., Chan, P., Cameron, P., Joynt, G. M., Ahuja, A., Yung, M. Y., Leung, C. B., To, K. F., Lui, S. F., Szeto, C. C., Chung, S. e Sung, J. J. Y. (2003). A Major Outbreak of Severe Acute Respiratory Syndrome in Hong Kong. New England Journal of Medicine, 348(20), 1986-1994.

Leydesdorff, L. e Bornmann, L. (2016). The operationalization of "fields" as WoS subject categories (WCs) in evaluative bibliometrics: The cases of "library and information science" and "science \& technology studies". Journal of the Association for Information Science and Technology, 67(3), 707-714.

Li, W., Moore, M. J., Vasilieva, N., Sui, J., Wong, S. K., Berne, M. A., Somasundaran, M., Sullivan, J. L., Luzuriaga, K., Greenough, T. C., Choe, H. e Farzan, M. (2003). Angiotensin-converting enzyme 2 is a functional receptor for the SARS coronavirus. Nature, 426(6965), 450-454.

Liu, C., Zhou, Q., Li, Y., Garner, L. V., Watkins, S. P., Carter, L. J., Smoot, J., Gregg, A. C., Daniels, A. D., Jervey, S. e Albaiu, D. (2020). Research and Development on Therapeutic Agents and Vaccines for COVID-19 and Related Human Coronavirus Diseases. ACS Central Science, 6(3), 315-331.

Malta, M., Rimoin, A. W. e Strathdee, S. A. (2020). The coronavirus 2019-nCoV epidemic: Is hindsight 20/20? EClinicalMedicine, 20.

Marra, M. A., Jones, S. J. M., Astell, C. R., Holt, R. A., Brooks-Wilson, A., Butterfield, Y. S. N., Khattra, J., Asano, J. K., Barber, S. A., Chan, S. Y., Cloutier, A., Coughlin, S. M., Freeman, D., Girn, N., Griffith, O. L., Leach, S. R., Mayo, M., McDonald, H., Montgomery, S. B., Pandoh, P. K., Petrescu, A. S., Robertson, A. G., Schein, J. E., Siddiqui, A., Smailus, D. E., Stott, J. M., Yang, G. S., Plummer, F., Andonov, A., Artsob, H., Bastien, N., Bernard, K., Booth, T. F., Bowness, D., Czub, M., Drebot, M., Fernando, L., Flick, R., Garbutt, M., Gray, M., Grolla, A., Jones, S., Feldmann, H., Meyers, A., Kabani, A., Li, Y., Normand, S., Stroher, U., Tipples, G. A., Tyler, S., Vogrig, R., Ward, D., Watson, B., Brunham, R. C., Krajden, M., Petric, M., Skowronski, D. M., Upton, C. e Roper, R. L. (2003). The Genome Sequence of the SARS-Associated Coronavirus. Science, 300(5624), 1399-1404.

National Institute of Allergy and Infectious Diseases. (2020). Coronaviruses 2020. Retrieved 02. Mar 2020, from https://www.niaid.nih.gov/diseases-conditions/coronaviruses?researchers=true

Peiris, J. S. M., Lai, S. T., Poon, L. L. M., Guan, Y., Yam, L. Y. C., Lim, W., Nicholls, J., Yee, W. K. S., Yan, W. W., Cheung, M. T., Cheng, V. C. C., Chan, K. H., Tsang, D. N. C., Yung, R. W. H., Ng, T. K. e Yuen, K. Y. (2003). Coronavirus as a possible cause of severe acute respiratory syndrome. The Lancet, 361(9366), 1319-1325.

Pinho, I. (2012). Knowledge Management in Research Centers - Barriers / Facilitators. University of Aveiro.

Pinho, I. e Diogo, S. (2018). Enhancing the Visibility and Impact of Scholarly Research: an exploratory study on knowledge production settings. Revista Meta: Avaliação, 10(30), 502.

Poutanen, S. M., Low, D. E., Henry, B., Finkelstein, S., Rose, D., Green, K., Tellier, R., Draker, R., Adachi, D., Ayers, M., Chan, A. K., Skowronski, D. M., Salit, I., Simor, A. E., Slutsky, A. S., Doyle, P. W., Krajden, M., Petric, M., Brunham, R. C. e McGeer, A. J. (2003). Identification of Severe Acute Respiratory Syndrome in Canada. New England Journal of Medicine, 348(20), 1995-2005.

Raj, V. S., Mou, H., Smits, S. L., Dekkers, D. H. W., Müller, M. A., Dijkman, R., Muth, D., Demmers, J. A. A., Zaki, A., Fouchier, R. A. M., Thiel, V., Drosten, C., Rottier, P. J. M., Osterhaus, A. D. M. E., Bosch, B. J. e Haagmans, B. L. (2013). Dipeptidyl peptidase 4 is a functional receptor for the emerging human coronavirus-EMC. Nature, 495(7440), 251-254. 
Rego, A., Pinho, I., Pedrosa, J. e Cunha, M. P. (2009). Barriers and facilitators to knowledge management in university research centers: an exploratory study. Management Research, 7(1), 33-47.

Rota, P. A., Oberste, M. S., Monroe, S. S., Nix, W. A., Campagnoli, R., Icenogle, J. P., Peñaranda, S., Bankamp, B., Maher, K., Chen, M.-h., Tong, S., Tamin, A., Lowe, L., Frace, M., DeRisi, J. L., Chen, Q., Wang, D., Erdman, D. D., Peret, T. C. T., Burns, C., Ksiazek, T. G., Rollin, P. E., Sanchez, A., Liffick, S., Holloway, B., Limor, J., McCaustland, K., Olsen-Rasmussen, M., Fouchier, R., Günther, S., Osterhaus, A. D. M. E., Drosten, C., Pallansch, M. A., Anderson, L. J. e Bellini, W. J. (2003). Characterization of a Novel Coronavirus Associated with Severe Acute Respiratory Syndrome. Science, 300(5624), 1394-1399.

Tsang, K. W., Ho, P. L., Ooi, G. C., Yee, W. K., Wang, T., Chan-Yeung, M., Lam, W. K., Seto, W. H., Yam, L. Y., Cheung, T. M., Wong, P. C., Lam, B., Ip, M. S., Chan, J., Yuen, K. Y. e Lai, K. N. (2003). A Cluster of Cases of Severe Acute Respiratory Syndrome in Hong Kong. New England Journal of Medicine, 348(20), 1977-1985.

Zaki, A. M., van Boheemen, S., Bestebroer, T. M., Osterhaus, A. D. M. E. e Fouchier, R. A. M. (2012). Isolation of a Novel Coronavirus from a Man with Pneumonia in Saudi Arabia. New England Journal of Medicine, 367(19), 1814-1820. 